\title{
Insect miscellany
}

Insects, Science and Society. (Proceedings of a Symposium held at Cornell University, Ithaca, New York, October 14-15, 1974.) Edited by David Pimentel. (Academic: New York and London, July 1975.) \$15; £7.20.

THE symposium on which this book is based was held in Cornell University in October 1974 to celebrate the centenary of the teaching of entomology at Cornell-indeed, in the United States-coupled with the name of the founding father John Henry Comstock, who gave the first course of lectures while still an undergraduate. The well known achievements of Comstock are briefly reviewed by E. H. Smith. D. Pimentel gives a general introduction on insects and crop production in the US, and J. J. McKelvey a colourful picture of 'insects and man'. E. O. Wilson contributes a brilliant short essay on the evolution of insect societies, in which their potential for studying the nature of sociality is stressed.

R. D. Alexander gives a lengthy account of chorussing behaviour in Orthoptera and cicadas, which is largely a re-interpretation of the author's earlier observations, the stress now being laid on the selective advantage secured by individual males in the competitive search for a mate, rather than on the advantage for the group. W. L. Roelofs reviews his own researches on the red-banded tortrix (leafroller) as an example of the physiological properties of pheromones and of their potential use in monitoring populations and perhaps in control by trapping or mating disruption. In a stimulating essay J. S. Kennedy argues persuasively for the view that all migration (and that includes 'dispersal') in insects involves a behavioural response, an essential component of which is the suppression of reproductive ('vegetative') activity and its temporary substitution by active locomotion, with or without passive transport by the elements. M. D. Pathak reviews the methods and results of plant breeding for resistance to insect pests as illustrated by the wide ranging studies on rice.

According to a valuable contribution by T. R. E. Southwood the competing models of population dynamics at varying densities all contain elements of the truth, and that an approximation to real life can be obtained by integrating these models into a single framework which also takes account of habitat stability. As P. S. Messenger points out in a paper on parasites, predators and population dynamics in California, that even in highly developed monocultures the complex of insect species is very large and general predators are a major element in integrated control schemes. W. Klassen gives an account of the planning and administration of the insect control programmes as organised by the US Department of Agriculture, in which it is claimed that increasing stress is being placed on 'integrated control'.

The final article, by L. D. Newsom, deals with 'pest management' (synonymous with integrated control)-the standard procedure of the entomologists of the nineteenth century, which he illustrates vividly in relation to cotton and soya bean culture in the US. There secms little doubt that the future lies with these ecological procedures. But they require skilled and imaginative entomologists; they are vigorously opposed by the salesmen of the chemical industry whose approach appeals more to the grower; and they still lack administrative encouragement. They do, however, have some enthusiasts on their side.

Although not closely integrated, and although reproduced in offset typescript, this book contains a high proportion of valuable articles.

V. B. Wigglesworth Stellar essays

Problems in Stellar Atmospheres and Envelopes. Edited by B. Baschek, W. H. Kegel and G. Traving. Pp. $\mathrm{xix}+375$. (Springer: Berlin and New York, 1974.) DM48; \$20.70.

THE occasion of the seventieth birthday of one of the world's most distinguished authorities on the theory of stellar atmospheres and the quantitative analysis of spectral linesProfessor Albrecht Unsöld of Kiel University-has been marked by the production of this Festschrift to which eleven of his former pupils have contributed review articles on stellar atmospheres and related topics, expressing at the same time the hope that others will find them interesting and stimulating.

This hope is certainly fulfilled by the great majority of the articles, in which the authors give their personal viewpoint on several topics of current interest in stellar astrophysics. Classical problems relating to the structure of stellar atmospheres are discussed by D. Labs, who gives a convincing defence of the solar irradiance tables published by Labs and Neckel in 1968 and 1970, and by Mrs E. Böhm-Vitense who uses a grid of model stellar atmospheres in radiative and local thermodynamic equilibrium to predict continuous spectra and colour indices as a function of temperature, luminosity and chemical composition. From a less classical point of view, G. Traving discusses the formation of spectral lines in turbulent media and the physical significance of the controversial empirical parameter known as "microturbulence". Moving out from "atmospheres" to "envelopes", W. H. Kegel describes the theory of maser action in $\mathrm{OH}$ and $\mathrm{H}_{2} \mathrm{O}$ emission sources, while L. Oster gives a rather entertaining historical review of radio emission from stars and its significance -a subject that has been close to Unsöld's heart since the 1950 s- - and K. H. Böhm describes physical conditions in the rather mysterious Herbig-Haro objects and in the related nebulae surrounding $T$ Tauri stars, which may result from shock-heating by the impact of a stellar wind on the intracloud medium.

The remaining articles concern the application of stellar atmosphere theory to abundances of elements and other problems in stellar internal structure and evolution. K. Hunger discusses the helium stars, concluding that some of the intermediate variety (as well as the extreme ones) achieve this state by nuclear evolution, whereas others may be affected by atmospheric diffusion. Further abundance anomalies in hot stars (mainly with weak helium lines) are lucidly described by $\mathbf{B}$. Baschek, together with the many mechanisms that have been proposed to account for them, among which the diffusion hypothesis of G. Michaud is generally the most successful, although other mechanisms may also be involved. Diffusion is also clearly important in white dwarfs, which are discussed in a useful review by $V$. Weidemann that covers their role in galactic evolution as well as their atmospheric properties. K. Kodaira describes the spectral analysis of A-type horizontal-branch stars which is of interest both as a source of information on the chemical composition of old obiects and as a means of determining the mass-luminosity ratio, which can then be compared with predictions from evolution and pulsation theories; and D. Reimers discusses the rate of mass loss from red giants on the basis of circumstellar absorption-line observations, deriving an empirical formula which shows that observed mass-loss rates are sufficient to satisfy evolutionary requirements for stars having less than twice the mass of the Sun.

Altogether this book is a worthy tribute to its illustrious dedicatee and a welcome addition to the astronomical review literature.
Bernard Pagel 